\title{
Investigating a pathogenic role for TXNDC5 in tumors
}

\author{
XIAOTIAN CHANG ${ }^{1}$, BING XU $^{1}$, LIN WANG $^{2}$, YAO WANG $^{1}$, YUEJIAN WANG $^{1}$ and SUHUA YAN ${ }^{1}$ \\ ${ }^{1}$ Medical Research Center of Shandong Provincial Qianfoshan Hospital, Shandong University, Jinan, Shandong 250014; \\ ${ }^{2}$ Research Center for Medicinal Biotechnology, Shandong Academy of Medical Sciences, \\ Jinan, Shandong 250062, P.R. China
}

Received June 29, 2013; Accepted August 2, 2013

DOI: 10.3892/ijo.2013.2123

\begin{abstract}
The expression of TXNDC5, which is induced by hypoxia, stimulates cell proliferation and angiogenesis. The increased cell proliferation, angiogenesis and hypoxia are main features of tumor tissues. The present study aimed to characterize the expression of TXNDC5 in various tumor types and to investigate the role of TXNDC5 in the growth, proliferation and migration of tumor cells. The study also determined susceptibility of TXNDC5 gene on tumor risk. The expression of TXNDC5 in tumor tissues was determined by immunohistochemistry using a tissue array that contained various types of tumor tissues. The expression levels of TXNDC5 in tumor tissues and healthy tissues were quantitatively analyzed using western blotting. Furthermore, HeLa cells and U2OS cells were treated with anti-TXNDC5 siRNA to knockdown the expression levels of TXNDC5 to study its role in cell proliferation and migration. The cell proliferation and migration of the transfected tumor cells were determined by MTT and Transwell migration assays, respectively. Ninety-six tag SNPs across the TXNDC5 locus were genotyped using custom-designed Illumina 384-SNP VeraCode microarrays. Our immunohistochemical staining revealed significant expression of TXNDC5 in breast invasive ductal carcinomas, cervical squamous cell carcinomas, esophageal squamous cell carcinomas, gastric carcinomas, hepatocellular carcinomas, ovarian papillary serous carcinomas, prostate cancers and undifferentiated cell carcinomas of the lung. Western blot analysis also detected significantly higher TXNDC5 expression in tumor tissues of breast cancers, gastric adenocarcinomas and rectal cancers compared to the adjacent healthy tissues. Decreased growth and invasive potential were observed in cultured HeLa cells and U2OS cells when TXNDC5 gene expression was knocked down. The case-control analysis showed a significant difference in allele frequency and genotype frequency for rs9505298,
\end{abstract}

Correspondence to: Professor Xiaotian Chang, Medical Research Center of Shandong Provincial Qianfoshan Hospital, Shandong University, Jingshi Road 16766, Jinan, Shandong 250014, P.R. China E-mail: changxt@126.com

Key words: TXNDC5, tumor, cell proliferation, cell migration, susceptibility rs7771314, rs2815128, rs13210097 and rs9392182 between cervical carcinoma, esophageal carcinoma and liver cancer patients and controls. These results suggest that TXNDC5 has increased expression in many tumors that is involved in the proliferation and migration of tumor cells, acting as a tumorenhancing gene. The study also suggests that TXNDC5 gene is susceptible to cervical carcinoma, esophageal carcinoma and liver cancer risk.

\section{Introduction}

Thioredoxin domain containing 5 (TXNDC5) protein, also named ERp46, has a protein disulfide isomerase (PDI) domain that exhibits a high sequence similarity to thioredoxin, a catalyst of the rate limiting reaction of disulfide bond formation, isomerization and reduction $(1,2)$. Yeast complementation tests showed that TXNDC5 can conduct PDI functions in vivo (3). Indirect immunofluorescence microscopy and subcellular fractionation studies have shown that TXNDC5 is present both in the endoplasmic reticulum and in the plasma membrane (4). TXNDC5 is highly expressed in endothelial cells during hypoxic conditions, and it plays important roles in anti-oxidative injury, anti-anoxia-induced apoptosis and the promotion of cell proliferation $(1,2)$.

Recent studies reported that TXNDC5 is overexpressed in some tumors including the cervix, uterus, stomach and lung cancers (5). Wang et al also found that TXNDC5 was significantly upregulated in colorectal cancer tissues compared with normal mucosa (6). Vincent et al demonstrated that $62 \%$ of non-small cell lung carcinomas exhibit an increase in TXNDC5 expression compared with normal lung tissue (7). TXNDC5 has been reported to be involved in cancer progression. Zhang et al inserted TXNDC5 cDNA into the gastric cell line HFE145. They demonstrated that TXNDC5 promotes growth, proliferation and invasion of gastric cells (8). Thus, TXNDC5 can be thought of as a tumor-enhancing gene. However, there is no comprehensive investigation reported on TXNDC5 expression in various tumor types, and not much is known about the functional roles of the TXNDC5 in the tumorigenic process.

In the present study, we investigated the expression of TXNDC5 in various tumors. We also investigated the role of TXNDC5 in cell proliferation and migration of cultured tumor cell lines using small interfering RNAs. In addition, we determined whether common polymorphisms in TXNDC5 
are associated with various tumors using microarray. To our knowledge, this is the first study to examine this potential association of the genes with tumors.

\section{Materials and methods}

Western blot analysis. Breast cancer $(\mathrm{n}=4)$, gastric adenocarcinoma $(n=4)$ and rectal cancer $(n=4)$ tissue samples were collected during excision surgery in Shandong Provincial Qianfoshan Hospital (Jinan, China). Parallel healthy tissues $5 \mathrm{~cm}$ from the tumors were also collected during the excision surgery. The tumor diagnosis was verified by histological methods, and pathological categorization was performed according to the World Health Organization (WHO) classification system. All patients signed informed consents, and this study was approved by the ethics committee of Shandong Provincial Qianfoshan Hospital.

Two hundred micrograms of the tumor tissues from gastric adenocarcinoma, rectal cancer, breast cancer and their parallel healthy tissues were homogenized in Cell Lysis Solution (Sigma) and centrifuged at $16,000 \mathrm{x}$ g for $5 \mathrm{~min}$ at $4^{\circ} \mathrm{C}$. The total protein was separated by sodium dodecyl sulfate-polyacrylamide gel electrophoresis (SDS-PAGE) and trans-blotted onto nitrocellulose membranes (Amersham, Piscataway, NJ, USA). Western blot analysis was conducted using antibodies against human TXNDC5 at a 2,000-fold dilution overnight at $4^{\circ} \mathrm{C}$. The anti-TXNDC5 antibodies that were used were raised in goats using the oligopeptide SLHRFVLSQAKDEL from TXNDC5. The western signals were visualized using the Protein Detector BCIP/NBT Western Blot kit (Beyotime, Haimen, China) following the manufacturer's instructions. A separate membrane that was prepared by the same protocol was probed with an anti-GAPDH antibody (Santa Cruz Biotechnology, Santa Cruz, CA, USA) to normalize the sample loading.

Immunohistochemistry. Tissue arrays were obtained commercially from Chaoying Bioscience (Shanxi, China). The array slides contained tumor and normal samples from each of the following tissues: breast $(n=208)$, cervical $(n=33)$, colon carcinoma $(n=50)$, esophageal cancer $(n=76)$, gastric carcinoma $(n=84)$, hepatocellular $(n=73)$, ovarian $(n=46)$, pancreatic cancer $(n=24)$, prostate cancer $(n=54)$, rectal $(n=29)$ and small cell lung $(n=100)$. Clinical data, including the age, sex, clinical pathological diagnosis, and origin of every participant, were provided by the manufacturer. Tissue sections were de-paraffinized and re-hydrated by standard procedures. Before the anti-TXNDC5 antibodies were applied, the tissue sections were heated at $95^{\circ} \mathrm{C}$ for $10 \mathrm{~min}$ in citrate buffer solution (Sigma) for antigen recovery followed by incubation with an endogenous peroxidase inhibitor (Maixin-Bio, Fuzhou, China) for $30 \mathrm{~min}$ at room temperature. After washing with PBS buffer $\left(\mathrm{NaCl} 0.132 \mathrm{M}, \mathrm{K}_{2} \mathrm{HPO}_{4} 0.0066 \mathrm{M}\right.$, $\mathrm{KH}_{2} \mathrm{PO}_{4} 0.0015 \mathrm{M}$ in distilled water, $\mathrm{pH} 7.6$ ), the sections were incubated with an antibody directed against TXNDC5 (Abcam) overnight at $4^{\circ} \mathrm{C}$. Immunoreactions were processed using the UltraSensitive ${ }^{\mathrm{TM}} \mathrm{S}-\mathrm{P}$ kit (Maixin-Bio) according to the manufacturer's instructions. Immunoreactive signals were visualized using the DAB substrate, which stains the target protein yellow. Cell structures were counterstained with hematoxylin.
To determine the antibody specificity and optimize the antibody dilution, the tissue samples were incubated with goat pre-immune serum (Maixin-Bio) or treated with the modification buffer without addition of antibody.

Inhibiting TXNDC5 expression in cultured tumor cell lines with siRNAs. HeLa cells (originally from human cervical cancer) and U2OS cells (originally from human osteosarcoma) were cultured in McCoy's 5A medium (Gibco-BRL, Carlsbad, CA, USA) supplemented with $100 \mathrm{U} / \mathrm{ml}$ penicillin, $100 \mathrm{mg} / \mathrm{ml}$ streptomycin (Gibco-BRL) and 15\% (v/v) fetal bovine serum (Gibco-BRL). siRNA oligonucleotides targeting TXNDC5 (target mRNA sequence: 5'-CACATACAGGCTTAAGCTCTA-3') were designed and synthesized by Qiagen (Hilden, Germany). Cultured tumor cells were transfected with $100 \mathrm{nM}$ of the siRNA using the HiPerFect transfection reagent (Qiagen) according to the manufacturer's protocol. The cells were harvested for analysis $48 \mathrm{~h}$ following the transfection. Mm/Hs-MAPK1 siRNA (AATGCTGACTCCAAAGCTCTG) and AllStars Negative Control siRNA, which were provided with the kit, were used as positive and negative controls, respectively, in parallel transfections. Mm/Hs_MAPK1 Control siRNA is a positive control targeting both the human and mouse MAPK1 genes. AllStars Negative Control siRNA is the most thoroughly tested and validated negative control siRNA.

Cell proliferation assay. U2OS and HeLa cells were seeded onto 96-well culture plates and incubated until they reached $80 \%$ confluence. The cultures were then treated with TXNDC5 siRNA as described above. An MTT [3-(4,5-dimethylthiazol2-yl)-2,5-diphenyltetrazolium bromide] assay was performed $48 \mathrm{~h}$ later by adding $100 \mu \mathrm{l}$ of $1 \mathrm{mg} / \mathrm{ml}$ MTT (Amresco, Solon, $\mathrm{OH}$, USA) mixed with culture media into each well and incubating for $4 \mathrm{~h}$ at $37^{\circ} \mathrm{C}$ in the dark. The MTT solution was then removed, and the cells were washed twice in PBS followed by air drying. The resulting MTT-formazan products were extracted with $100 \mu \mathrm{l}$ DMSO in the dark at room temperature, and their absorbance was measured at $490 \mathrm{~nm}$ with a spectrophotometer. The data were obtained from three independent experiments.

Cell migration assay. A cell migration assay was conducted in a Transwell apparatus (Costar Corning, Corning, NY, USA). Cells were seeded in the upper compartment of the Transwell apparatus while the lower compartment was filled with DMEM with $10 \%$ FBS, and the plates were incubated at $37^{\circ} \mathrm{C}$ for $24 \mathrm{~h}$ until the cells reached $80 \%$ confluence. At this point, the cells were transfected with $100 \mathrm{nM}$ of TXNDC5 siRNA, and the cells were incubated for an additional $48 \mathrm{~h}$ in the incubator. The upper surface of the insert was wiped with cotton swabs to remove non-invading cells, and the bottom surface of the insert was stained with Giemsa. The number of cells that invaded through the membrane was counted in 5 random fields at x100 magnification. The data were obtained from three independent experiments.

Statistical analysis of the data was performed using the SPSS V.16 software (SPSS, Chicago, IL, USA). Multiple comparisons were conducted with ANOVA, and a t-test was used to assess significant differences between two groups. P-values that were $<0.05$ were considered significant. The errors in the data are shown as standard deviation (SD). 
Table I. SNPs selected for illumina microarray.

\begin{tabular}{|c|c|c|c|c|}
\hline $\begin{array}{l}\text { Locus } \\
\text { name }\end{array}$ & $\begin{array}{c}\text { Gene } \\
\text { symbol }\end{array}$ & Coordinate & Location & $\begin{array}{c}\text { Coding } \\
\text { status }\end{array}$ \\
\hline rs 1044104 & BMP6/TXNDC5 & 7881311 & COMPLEX & \\
\hline rs9505298 & BMP6/TXNDC5 & 7881449 & COMPLEX & \\
\hline rs41302895 & TXNDC5/BMP6 & 7881754 & COMPLEX & \\
\hline rs1043784 & TXNDC5/BMP6 & 7881931 & COMPLEX & \\
\hline rs7764128 & BMP6/TXNDC5 & 7882205 & COMPLEX & \\
\hline rs8643 & BMP6/TXNDC5 & 7883073 & COMPLEX & \\
\hline rs9502656 & TXNDC5 & 7883386 & CODING & NONSYN \\
\hline rs35264740 & TXNDC5 & 7883865 & INTRON & \\
\hline rs35871461 & TXNDC5 & 7884291 & INTRON & \\
\hline rs2277105 & TXNDC5 & 7884652 & CODING & NONSYN \\
\hline rs35126514 & TXNDC5 & 7885048 & INTRON & \\
\hline rs1225936 & TXNDC5 & 7885184 & INTRON & \\
\hline rs1225937 & TXNDC5 & 7885302 & INTRON & \\
\hline rs11758961 & TXNDC5 & 7885797 & INTRON & \\
\hline rs 1225938 & TXNDC5 & 7886534 & INTRON & \\
\hline rs454654 & TXNDC5 & 7886639 & INTRON & \\
\hline rs11962800 & TXNDC5 & 7886905 & INTRON & \\
\hline rs9505301 & TXNDC5 & 7887131 & INTRON & \\
\hline rs372578 & TXNDC5 & 7887223 & INTRON & \\
\hline rs7740689 & TXNDC5 & 7888066 & INTRON & \\
\hline rs89715 & TXNDC5 & 7888168 & INTRON & \\
\hline rs7745225 & TXNDC5 & 7888251 & INTRON & \\
\hline rs378963 & TXNDC5 & 7888328 & INTRON & \\
\hline rs45441296 & TXNDC5 & 7889033 & CODING & NONSYN \\
\hline rs34782746 & TXNDC5 & 7889254 & INTRON & \\
\hline rs7746818 & TXNDC5 & 7889466 & INTRON & \\
\hline rs71559189 & TXNDC5 & 7889796 & CODING & NONSYN \\
\hline rs60084141 & TXNDC5 & 7889894 & INTRON & \\
\hline rs1225947 & TXNDC5 & 7890121 & INTRON & \\
\hline rs 13873 & TXNDC5 & 7891160 & INTRON & \\
\hline rs73365786 & TXNDC5 & 7891230 & INTRON & \\
\hline rs7771314 & TXNDC5 & 7891403 & INTRON & \\
\hline rs34599679 & TXNDC5 & 7891514 & INTRON & \\
\hline rs1225949 & TXNDC5 & 7891673 & INTRON & \\
\hline rs3734589 & TXNDC5 & 7891775 & INTRON & \\
\hline rs9502658 & TXNDC5 & 7891947 & CODING & SYNON \\
\hline rs35365768 & TXNDC5 & 7892037 & INTRON & \\
\hline rs 1225950 & TXNDC5 & 7892143 & INTRON & \\
\hline rs11759946 & TXNDC5 & 7892360 & INTRON & \\
\hline rs72829238 & TXNDC5 & 7892575 & INTRON & \\
\hline rs7749719 & TXNDC5 & 7894695 & INTRON & \\
\hline rs58711083 & TXNDC5 & 7895348 & CODING & NONSYN \\
\hline rs443861 & TXNDC5 & 7896491 & INTRON & \\
\hline rs369086 & TXNDC5 & 7898875 & INTRON & \\
\hline rs408014 & TXNDC5 & 7899394 & INTRON & \\
\hline rs13218143 & TXNDC5 & 7899573 & INTRON & \\
\hline rs383084 & TXNDC5 & 7899657 & INTRON & \\
\hline rs1225954 & TXNDC5 & 7900028 & INTRON & \\
\hline rs1225955 & TXNDC5 & 7900709 & INTRON & \\
\hline rs6933089 & TXNDC5 & 7900856 & INTRON & \\
\hline rs13209404 & TXNDC5 & 7909967 & INTRON & \\
\hline rs13210097 & TXNDC5/MUTED & 7911345 & INTERGENIC & \\
\hline rs9502663 & TXNDC5/MUTED & 7911474 & INTERGENIC & \\
\hline rs3812162 & TXNDC5/MUTED & 7911702 & INTERGENIC & \\
\hline rs34066135 & MUTED/TXNDC5 & 7911855 & INTERGENIC & \\
\hline rs72829251 & TXNDC5/MUTED & 7911982 & INTERGENIC & \\
\hline rs1632346 & TXNDC5/MUTED & 7913546 & INTERGENIC & \\
\hline rs1743634 & TXNDC5/MUTED & 7916207 & INTERGENIC & \\
\hline rs9505309 & MUTED/TXNDC5 & 7917528 & INTERGENIC & \\
\hline rs6922018 & MUTED/TXNDC5 & 7918311 & INTERGENIC & \\
\hline rs6923488 & MUTED/TXNDC5 & 7918405 & INTERGENIC & \\
\hline
\end{tabular}

Table I. Continued.

\begin{tabular}{|c|c|c|c|c|}
\hline $\begin{array}{l}\text { Locus } \\
\text { name }\end{array}$ & $\begin{array}{l}\text { Gene } \\
\text { symbol }\end{array}$ & Coordinate & Location & $\begin{array}{c}\text { Coding } \\
\text { status }\end{array}$ \\
\hline rs1594467 & TXNDC5/MUTED & 7920361 & INTERGENIC & \\
\hline rs419588 & MUTED/TXNDC5 & 7920808 & INTERGENIC & \\
\hline rs365936 & MUTED/TXNDC5 & 7920904 & INTERGENIC & \\
\hline rs 1237879 & TXNDC5/MUTED & 7932261 & INTERGENIC & \\
\hline rs627957 & MUTED/TXNDC5 & 7936475 & INTERGENIC & \\
\hline rs155487 & MUTED/TXNDC5 & 7938773 & INTERGENIC & \\
\hline rs 10484327 & TXNDC5/MUTED & 7942566 & INTERGENIC & \\
\hline rs7764884 & MUTED/TXNDC5 & 7970540 & INTERGENIC & \\
\hline rs7763447 & MUTED/TXNDC5 & 7973380 & INTERGENIC & \\
\hline rs4959462 & MUTED/TXNDC5 & 7975135 & INTERGENIC & \\
\hline rs6597292 & TXNDC5/MUTED & 7975259 & INTERGENIC & \\
\hline rs197119 & TXNDC5/MUTED & 7976745 & INTERGENIC & \\
\hline rs6597293 & TXNDC5/MUTED & 7987883 & INTERGENIC & \\
\hline rs11754300 & TXNDC5/MUTED & 7988766 & INTERGENIC & \\
\hline rs7744601 & TXNDC5/MUTED & 7988910 & INTERGENIC & \\
\hline rs2567226 & MUTED/TXNDC5 & 7993977 & INTERGENIC & \\
\hline rs12204273 & TXNDC5/MUTED & 8002705 & INTERGENIC & \\
\hline rs9392182 & MUTED/TXNDC5 & 8009035 & INTERGENIC & \\
\hline rs2207720 & MUTED & 8019197 & INTRON & \\
\hline rs9392189 & MUTED & 8021532 & INTRON & \\
\hline rs 2815128 & MUTED & 8023462 & INTRON & \\
\hline rs2815142 & MUTED & 8043546 & INTRON & \\
\hline rs2743992 & MUTED & 8054722 & INTRON & \\
\hline rs2294436 & MUTED & 8057688 & INTRON & \\
\hline rs2743991 & MUTED & 8060175 & INTRON & \\
\hline rs12663430 & MUTED & 8060534 & INTRON & \\
\hline rs7763203 & MUTED & 8061872 & INTRON & \\
\hline rs9405369 & MUTED & 8062437 & INTRON & \\
\hline rs12207627 & MUTED & 8062532 & INTRON & \\
\hline rs2743989 & MUTED & 8064035 & INTRON & \\
\hline rs2743987 & MUTED & 8064303 & INTRON & \\
\hline rs35991100 & EEF1E1/MUTED & 8064677 & INTERGENIC & \\
\hline rs9328453 & MUTED/EEF1E1 & 8065127 & INTERGENIC & \\
\hline rs2815155 & MUTED/EEF1E1 & 8065230 & INTERGENIC & \\
\hline rs12660697 & EEF1E1/MUTED & 8065707 & INTERGENIC & \\
\hline rs7751386 & MUTED/EEF1E1 & 8066414 & INTERGENIC & \\
\hline
\end{tabular}

Genomic DNA isolation, SNP selection. Genomic DNA was extracted from whole blood samples with the Omega E-Z 96 Blood DNA kit (Omega Bio-Tek, Norcross, GA, USA) according to the manufacturer's protocol. After extraction, the genomic DNA was diluted to a final concentration of $15-20 \mathrm{ng} / \mu \mathrm{l}$ for the genotyping assays.

Tag single nucleotide polymorphisms (tagSNPs) across the TXNDC5 gene were determined by searching the HapMap database. Only SNPs with minor allele frequency greater (MAF) than $5 \%$ with a pair-wise $\mathrm{r}^{2} \geq 0.8$ were considered. Ninty-six SNPs that span 185,000 bases of the chromosome 6 were selected. The SNP information including locus name, gene symbol, coordinate, location and coding status is shown in Table I.

Genotyping using Illumina 384-SNP VeraCode microarray. We performed genotyping using a custom-designed Illumina 384-SNP VeraCode microarray (Illumina). Peripheral blood samples were collected from patients with breast cancer $(\mathrm{n}=281 ; 281$ female; aged 26-80 years; mean, 49.3 years), 

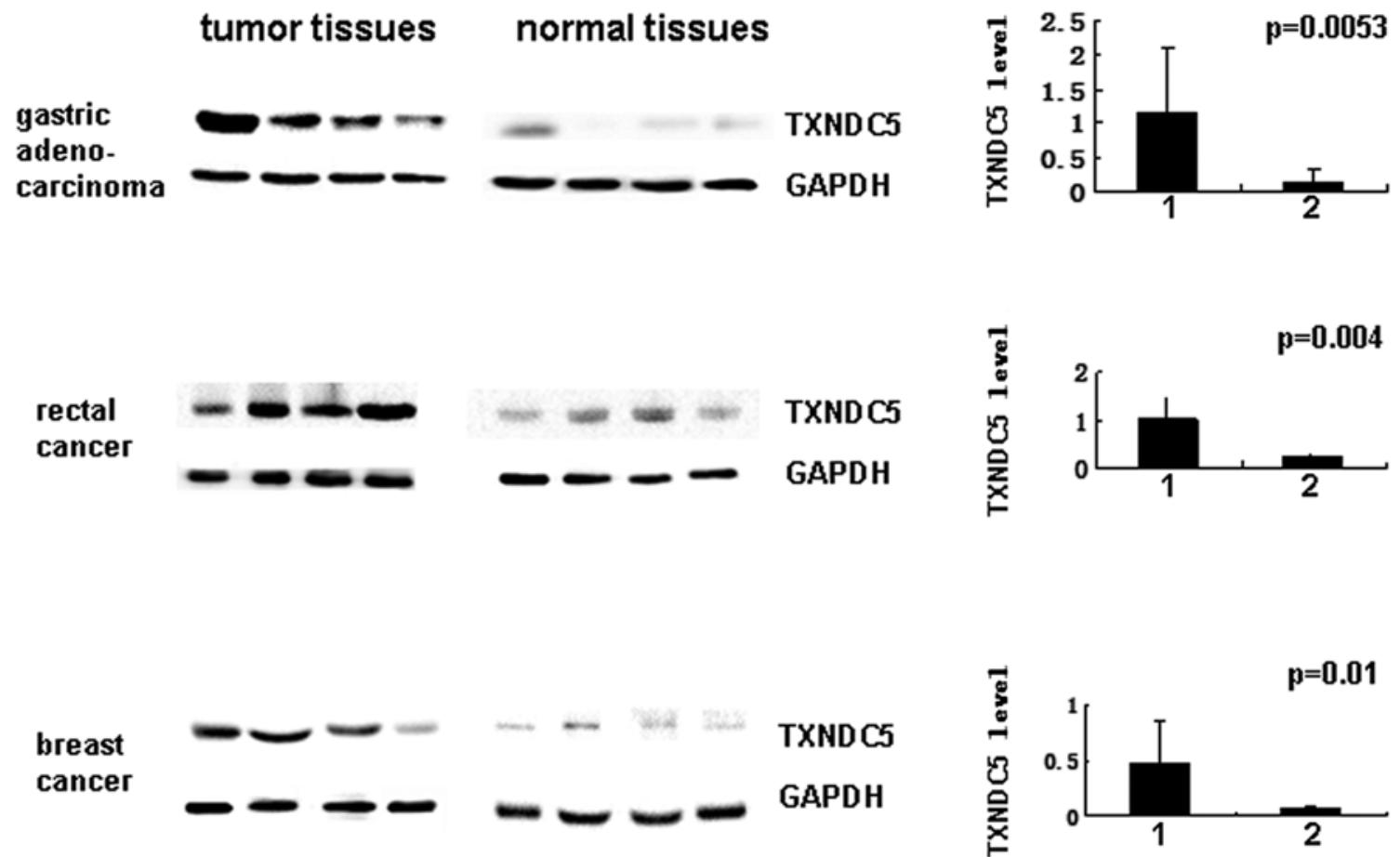

Figure 1. Quantitative analysis of TXNDC5 expression using western blot analysis. TXNDC5, which has a molecular weight of $50 \mathrm{kDa}$, was detected in (1) various tumor tissues and (2) their parallel normal tissues. Sample loading was normalized using GAPDH, which has a molecular weight of $37 \mathrm{kDa}$. TXNDC5 had significantly increased expression in gastric adenocarcinoma, rectal cancer and breast cancer tissues.

cervical carcinoma ( $\mathrm{n}=197 ; 197$ female; aged 29-61 years; mean, 54.4 years), esophageal carcinoma $(\mathrm{n}=221 ; 30$ female; aged 39-86 years; mean, 60.8 years), gastric carcinoma ( $n=308$; 64 female; aged 31-80 years; mean 58.2 years) and liver cancer $(\mathrm{n}=202 ; 30$ female; aged $30-79$ years; mean, 55.9 years). A total of 374 (125 female, aged 24-58 years) healthy individuals with a mean age of 40.2 years were blood donors. All patients signed informed consents, and this study was approved by the ethics committee of Shandong Provincial Qianfoshan Hospital. The genotyping was conducted with the BeadXpress Reader using the Illumina VeraCode GoldenGate Assay kit. A total of $500 \mathrm{ng}$ of sample DNA were used per assay. Genotype clustering and calling were performed using BeadStudio software (Illumina). This study was completed at Beijing Institute of Genomics of Chinese Academy of Sciences, which provided technical service for the genotyping.

The genotyping quality was examined by a detailed quality control procedure consisting of a $>95 \%$ successful call rate, duplicate calling of the genotypes, internal positive control samples and Hardy-Weinberg Equilibrium (HWE) testing. The SNPs were analyzed for association by comparing the MAF between the cases and controls. Dominant and recessive models were considered with respect to the minor allele. Associations of the SNPs with the diseases were evaluated using odds ratios (OR) with 95\% confidence intervals (CI). Fisher's exact test was used for comparisons between categorical variables. P-values $<0.05$ were considered to be statistically significant. Genotypic associations were assessed using Plink v1.07 (http://pngu.mgh. harvard.edu/purcell/plink/) and SHEsis (http://analysis.bio-x. $\mathrm{cn} /$ myAnalysis.php) software $(9,10)$. Multiple-test correction, including genomic-control correction, Bonferroni single-step correction, Holm step-down correction and Sidak single-step correction, were performed by Plink v1.07. Linkage disequilibrium (LD), coefficient ( $\mathrm{D}^{\prime}$ and $\mathrm{r}^{2}$ ) and haplotype were estimated by software Haploview 4.2 (http://www.broad.mit.edu/mpg/ haploview/) software (11).

\section{Results}

TXNDC5 expression in various tumor tissues. Western blots revealed a protein with a molecular weight of $50 \mathrm{kDa}$ in each of the tumor tissues and parallel normal tissues. The expression level was normalized using GAPDH as a reference. Compared with the expression in the parallel normal tissues, significantly increased TXNDC5 expression was detected in the gastric adenocarcinoma, rectal cancer and breast cancer tissues. These results were consistently observed in all the samples of these tumor types. The results are shown in Fig. 1.

Immunohistochemistry was used to detect the expression of TXNDC5 in a panel of tumor tissues. TXNDC5 was significantly expressed in the tumor tissues from breast invasive ductal carcinomas $(\mathrm{n}=170)$. The immuno-signal was observed in both tumor cells and mesenchymal cells in these tissues. TXNDC5 was not detected in the corresponding normal tissues except in some mesenchymal cells $(\mathrm{n}=38)$. TXNDC5 was significantly expressed in the tumor tissues from cervical squamous cell carcinomas $(n=22)$. The immuno-signal was observed in both tumor cells and mesenchymal cells in these tissues. TXNDC5 was not detected in the parallel normal tissues $(n=6)$ and tissues from cervical adenocarcinomas $(n=2)$ except in some mesenchymal cells. The expression of TXNDC5 was also observed in mesenchymal cells from the chronically inflamed mucosa 


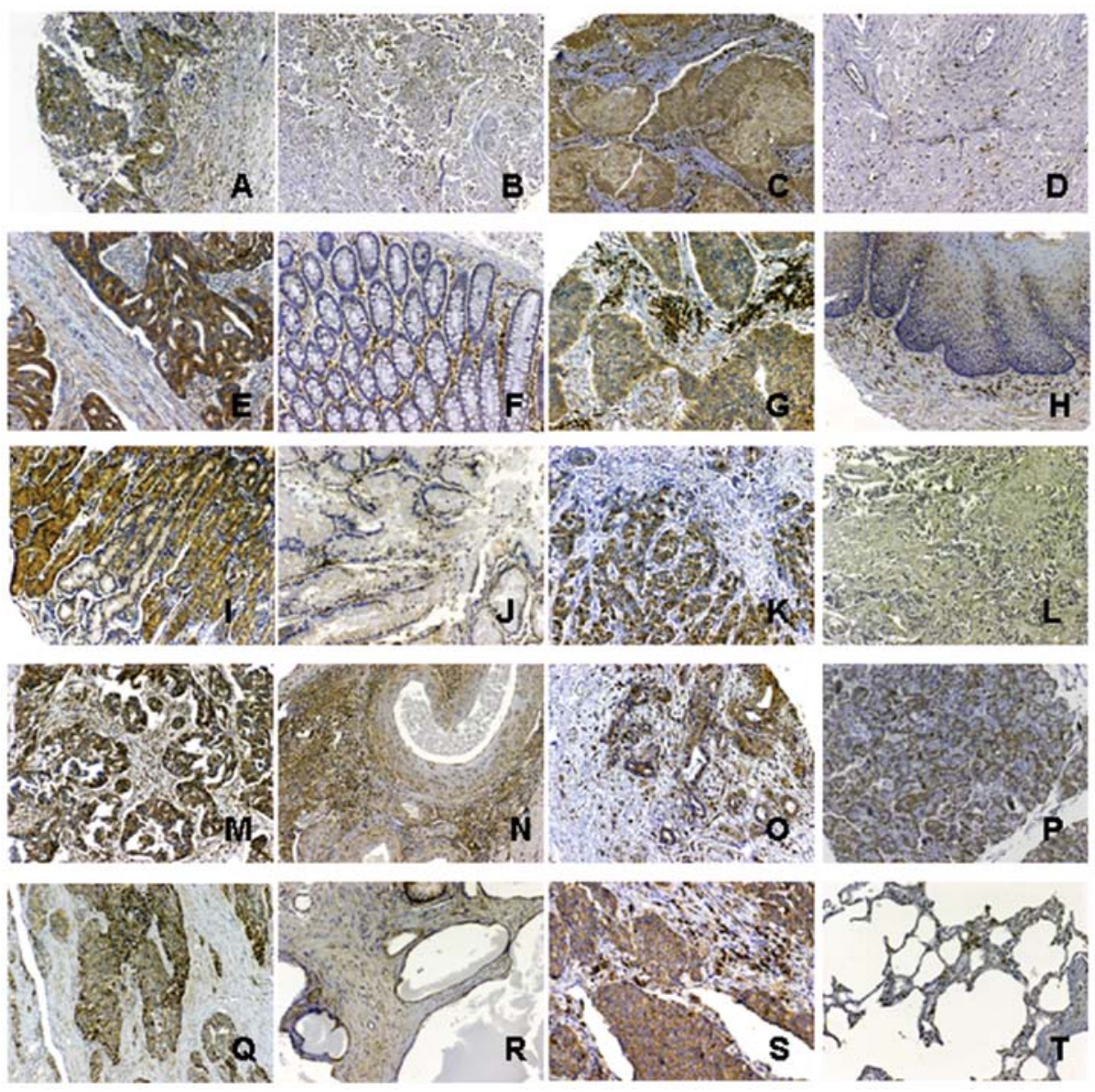

Figure 2. Immuno-location of TXNDC5 expression in various malignant tumors, non-tumor inflamed tissues and healthy tissues. The tissue structures were defined by counterstaining with hematoxylin. The representative images shown here are of: (A) breast invasive ductal carcinoma and (B) normal breast tissues; (C) cervical squamous cell carcinoma and (D) normal cervical tissues; (E) colon carcinoma and (F) normal colon tissues; (G) esophageal squamous cell carcinoma and (H) normal esophageal tissues; (I) gastric carcinoma and (J) normal gastric tissues; (K) hepatocellular carcinoma and (L) normal liver tissues; $(\mathrm{M})$ ovarian papillary serous carcinoma and $(\mathrm{N})$ normal ovarian tissues; $(\mathrm{O})$ pancreatic cancer and $(\mathrm{P})$ normal pancreatic tissues; $(\mathrm{Q})$ prostate cancer and (R) normal prostate tissues; (S) undifferentiated cell carcinoma of lung and (T) normal lung tissues. Original magnification, x100.

of the uterus, but the signal density was relative low $(\mathrm{n}=3)$. TXNDC5 was significantly expressed in the tumor tissues from colon carcinomas $(n=18)$ and rectal cancers $(n=23)$. Normal tissue from the colon $(n=32)$ and the rectum $(n=6)$ also showed TXNDC5 expression. The protein was expressed in both the tumor cells and mesenchymal cells, but the number of TXNDC5positive mesenchymal cells was relatively low in the normal tissues. TXNDC5 expression was detected in tissues from esophageal squamous cell carcinomas $(n=38)$ but not in healthy esophageal tissue $(\mathrm{n}=28)$. Its expression was also very strong in mesenchymal cells from the chronically inflamed mucosa of the esophagus $(\mathrm{n}=10)$. TXNDC5 expression was detected in gastric carcinoma $(n=43)$. The expression was present in both the tumor cells and mesenchymal cells of the tumor tissues. TXNDC5 expression was also detected in mesenchymal cells from the inflamed gastric mucosa $(n=23)$. TXNDC5 was not detected in healthy gastric tissues except in some mesenchymal cells $(n=18)$. TXNDC5 was detected in hepatocellular carcinomas $(n=51)$, but it was not present in samples from patients with chronic hepatitis cirrhosis $(n=22)$. TXNDC5 was significantly expressed in tissues from ovarian papillary serous carcinomas $(\mathrm{n}=14)$, ovarian endometrioid adenocarcinomas $(n=6)$ and ovarian clear cell carcinomas $(n=2)$. Expression was detected in both tumor cells and mesenchymal cells of these tumor tissues. TXNDC5 was also detected in some mesenchymal cells of normal ovary tissues $(n=24)$. TXNDC5 was significantly expressed in tumor cells and mesenchymal cells from carcinomas of the prostate $(\mathrm{n}=48)$. TXNDC5 expression was also detected in some mesenchymal cells and endothelial cells from prostates undergoing hyperplasia $(n=6)$. TXNDC5 was expressed in tumor cells and mesenchymal cells from pancreatic cancers $(n=12)$. The expression was also detected in cells of the normal pancreatic tissues $(n=12)$. TXNDC5 showed strong expression in tumor cells and mesenchymal cells from undifferentiated cell carcinomas of the lung $(n=80)$. TXNDC5 expression was also detected in some mesenchymal cells of normal lung tissues $(n=20)$. Immunostaining of TXNDC5 was observed in the cytoplasm of tumor cells and mesenchymal cells of both tumor tissues and the health tissues. These immunohistochemical results are shown in Fig. 2.

Cell proliferation, migration and invasion in the presence of TXNDC5 siRNA. Cultured HeLa and U2OS cells were transfected with $100 \mathrm{nM}$ siRNA. TXNDC5 expression in transfected U2OS was decreased considerably compared with untransfected cells. TXNDC5 expression was also mildly decreased 

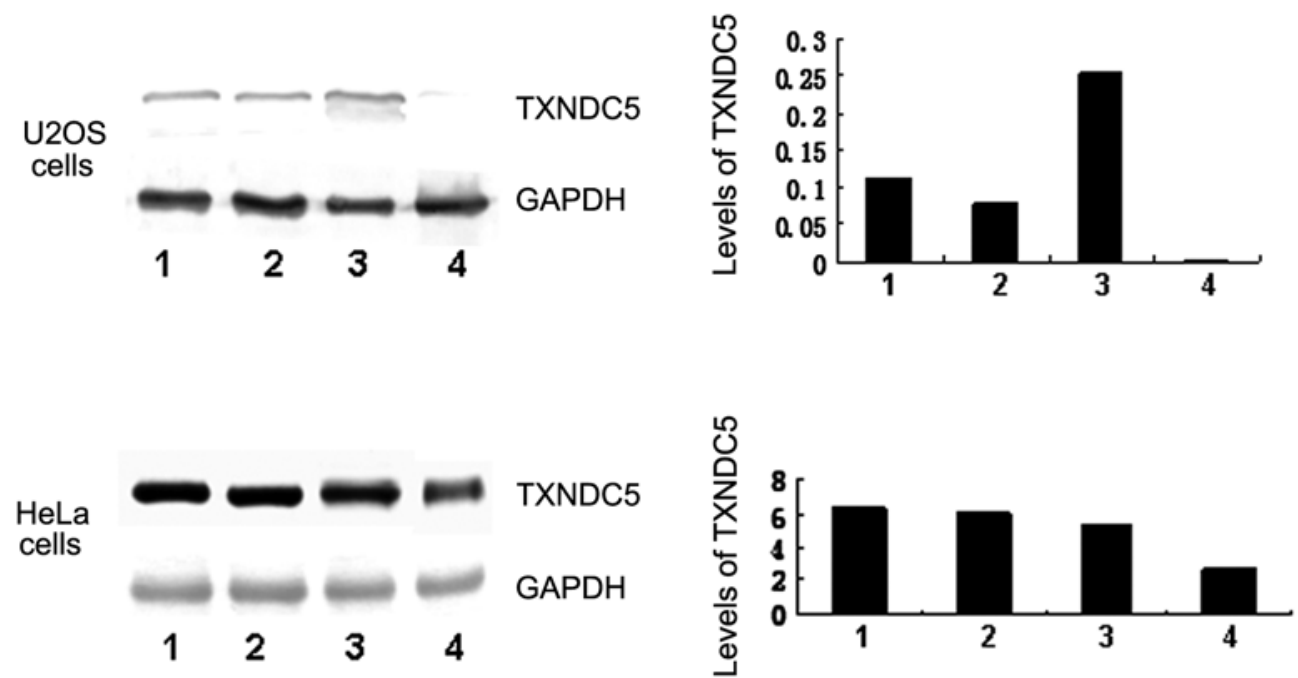

Figure 3. TXNDC5 expression in TXNDC5 targeted siRNA-treated U2OS and HeLa cells. The cultured tumor cell lines were transiently transfected with TXNDC5 targeted siRNA, and the effects of TXNDC5 knockdown were determined by western blot analysis. TXNDC5 expression was semi-quantitatively analyzed by normalizing the signal intensity of TXNDC5 to that of GAPDH. 1, positive control; 2, negative control; 3, mock; and 4, TXNDC5 siRNA treated.

in the siRNA-transfected HeLa cells. TXNDC5 levels were not significantly changed in the positive controls, negative controls or untransfected cells. The result is seen in Fig. 3.

A cell proliferation assay was performed using the U2OS cells and HeLa cells that were transfected with TXNDC5 siRNA. U2OS cells transfected with 100 nM TXNDC5 targeted siRNA exhibited a significant decrease in cell proliferation when compared with the positive and negative controls. Cell proliferation was mildly decreased in HeLa cells transfected with TXNDC5 targeted siRNA compared with untransfected cells. The data shown were obtained from three independent experiments. The result is shown in Fig. 4.

The migratory ability of U2OS cells and HeLa cells was examined using a 2-compartment Transwell system. Migration of both cell lines was significantly decreased when TXNDC5 expression was suppressed by TXNDC5 siRNA transfection. The data shown here were obtained from three independent experiments. The result is shown in Fig. 5.

Genotyping SNPs located in TXNDC5. Ninety-seven SNPs across TXNDC5 gene were genotyped using illumina microarray. All of the SNPs yielded genotypic data, and the study sample success rate was $98.1 \%$. SNPs except rs1225938, rs408014, rs1225954, rs2207720 and rs2743989 were in Hardy-Weinberg equilibrium $(\mathrm{P}>0.05)$ within the health samples. SNPs rs9502656, rs35264740, rs35871461, rs2277105, rs35126514, rs11758961, rs7740689, rs34782746, rs7746818, rs73365786, rs34599679, rs9502658, rs35365768, rs7749719, rs13218143, rs6933089, rs9502663, rs34066135, rs35991100, rs9328453 and rs7751386 did not show polymorphisms in the studied subjects. The differences in allele and genotype frequencies between the cases and controls were compared. The case-control analysis showed a significant difference in allele frequency and genotype frequency for rs 41302895 between breast cancer patients and health controls. The allele frequency and genotype frequency for rs9505298, rs41302895, rs7771314 and rs155487 provided statistically significant evidence for
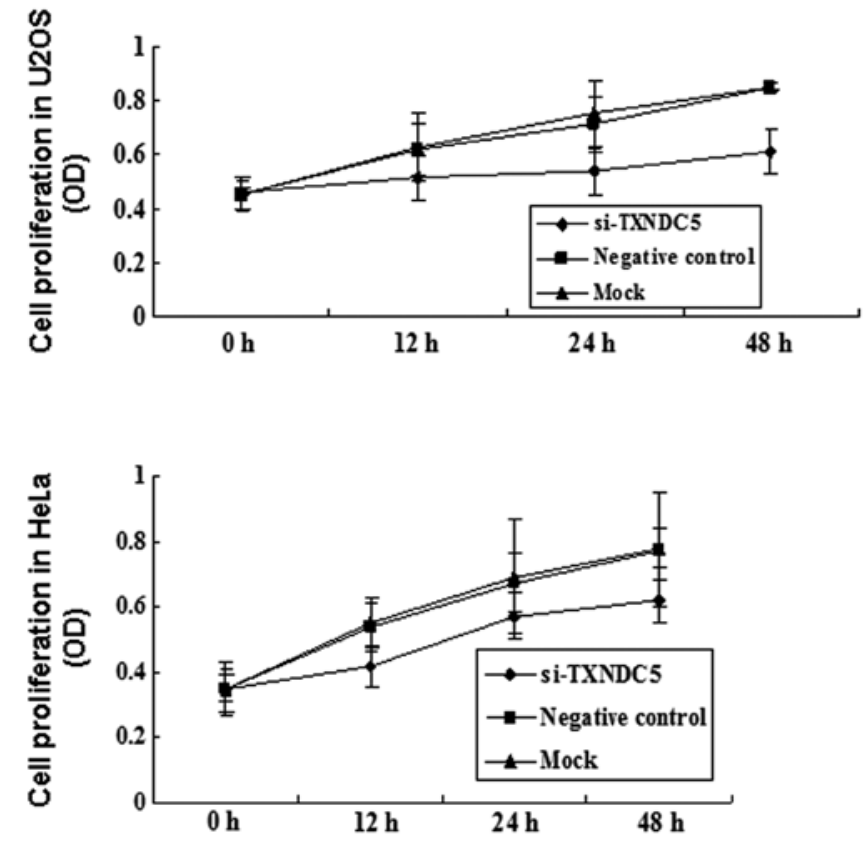

Figure 4. Proliferation of U2OS and HeLa cells treated with TXNDC5 targeted siRNA. Cell proliferation was determined using an MTT assay.

an association with cervical carcinoma. The case-control analysis showed a significant difference in allele frequency and genotype frequency for rs 1225950 and rs 2815128 between esophageal carcinoma patients and health controls. The analysis also showed a significant difference in allele frequency and genotype frequency for rs13210097 and rs9392182 between liver cancer patients and the controls. In addition, the allele frequency for rs383084, rs1632346, rs1237879 and rs11754300 provided statistically significant evidence for an association with cervical carcinoma, esophageal carcinoma, gastric carcinoma and liver cancer, respectively. The genotype frequency 

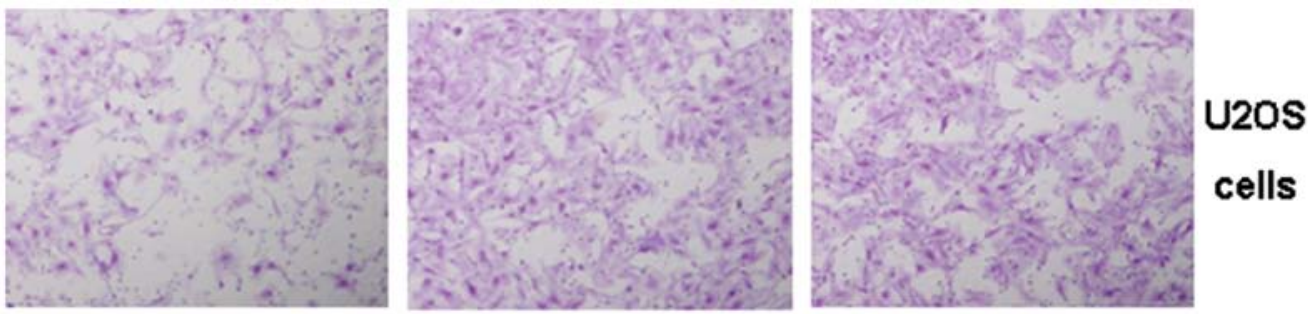

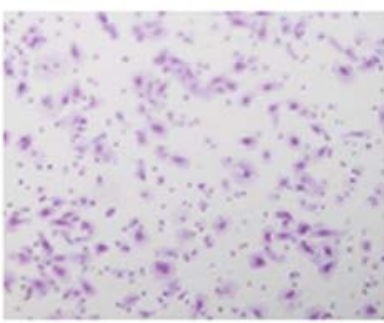

siRNA-TXNDC5

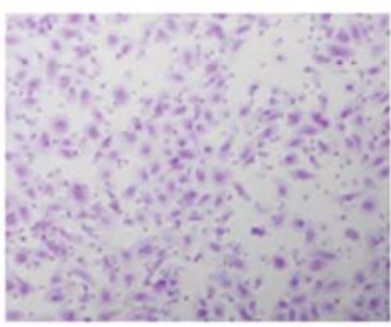

negative control

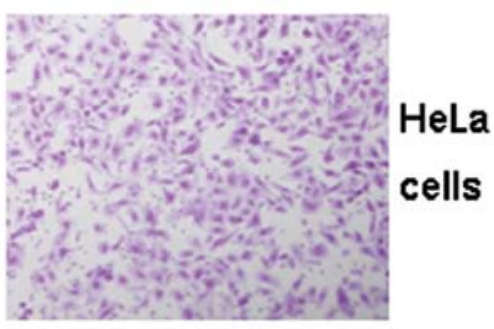

mock

Figure 5. Migration of U2OS and HeLa cells treated with TXNDC5 targeted siRNA. The migratory ability of the cultured cells was examined using a 2-compartment Transwell system. Crystal violet staining of the lower surface of the filters shows the number of cells that passed through the filter and attached to the lower side of the filter (x100).

for rs13873, rs1632346, rs9505309, rs1594467 and rs2815142 provided statistically significant evidence for an association with esophageal carcinoma, gastric carcinoma and breast cancer. Following multiple-test correction, these SNPs rs9505298 and rs7771314 still had significant difference in allelic frequency and genotypic frequency between cervical carcinoma patients and the controls; SNPs rs1632346, rs9505309, rs2815128 and rs2815142 still had significant difference in allelic frequency and genotypic frequency between esophageal carcinoma patients and the controls; SNPs rs13210097, rs11754300, rs9392182 and rs2815128 still had significant difference in allelic frequency and genotypic frequency between liver cancer patients and the controls. The above result is shown in Table II. The Illumina microarray data has been submitted to NCBI Gene Expression Omnibus (GEO), a public functional genomics data repository supporting MIAME-compliant data submissions. The record was approved and assigned GEO accession number (GSE39428).

\section{Discussion}

In the present study, immunohistochemistry detected significant expression of TXNDC5 in breast invasive ductal carcinoma, cervical squamous cell carcinoma, esophageal squamous cell carcinomas, gastric carcinoma, hepatocellular carcinoma, ovarian papillary serous carcinoma, prostate cancer and undifferentiated cell carcinoma of the lung tissues. TXNDC5 expression was also quantitatively assessed by western blot analysis. In comparison with parallel normal tissues, TXNDC5 expression was significantly increased in the tumor tissues of breast cancers, gastric adenocarcinomas and rectal cancers. This observation is in accordance with the results of immunohistochemistry, showing that TXNDC5 expression is increased in many tumor tissues. This is the first comprehensive investigation of TXNDC5 expression in various tumor types.
Tumors are thought to have decreased supply of oxygen, leading to hypoxia and hypo-perfusion $(12,13)$. TXNDC5 expression is upregulated by hypoxia and has a protective effect on endothelial cells by inducing the activities of chaperones important for protein folding of hypoxia-induced anti-apoptotic molecules $(1,2)$. In this study, HeLa and U2OS cells were treated with anti-TXNDC5 siRNA. Decreased growth and migration of these cells were observed when TXNDC5 expression was knocked down. This result suggests that TXNDC5 is involved in the proliferative and migration of tumor cells. Zhang et al also reported that TXNDC5 had a strong effect on gastric cell proliferation and could enhance the invasive capability of gastric cancer cells (8). We recently cultured synovial fibroblasts from patients with rheumatoid arthritis and incubated the cells with TXNDC5-siRNA or $\mathrm{CoCl}_{2}$, a chemical inducing hypoxia. Increased cell proliferation, cell migration and TXNDC5 expression were observed in the synovial fibroblasts following incubation with $1 \mu \mathrm{M}$ $\mathrm{CoCl} 2$; however, this effect was decreased when TXNDC5 expression was inhibited with $100 \mathrm{nM}$ siRNA (14). Together, these data suggest that hypoxia induces TXNDC5 expression and that inhibition of TXNDC5 expression prevents hypoxiainduced cell proliferation and migration.

TXNDC5 has been genetically mapped to chromosome 6p24.3. The gene encoding TXNDC5 is approximately $845.2 \mathrm{kbp}$, and it is divided into 13 exons. By genotyping 97 tagSNPs located in the TXNDC5 region, illumina microarray demonstrated significant association between TXNDC5 DNA polymorphisms and susceptibility to cervical carcinoma, esophageal carcinoma and liver cancer in a Chinese population. This is the first report to demonstrate the genetic effect of TXNDC5 on the tumor risk. To determine whether variations in the TXNDC5 gene contributed to the risk of developing non-segmental vitiligo, Jeong et al conducted a case-control association study within a Korean population. They genotyped seven SNPs and found that three exonic SNPs 


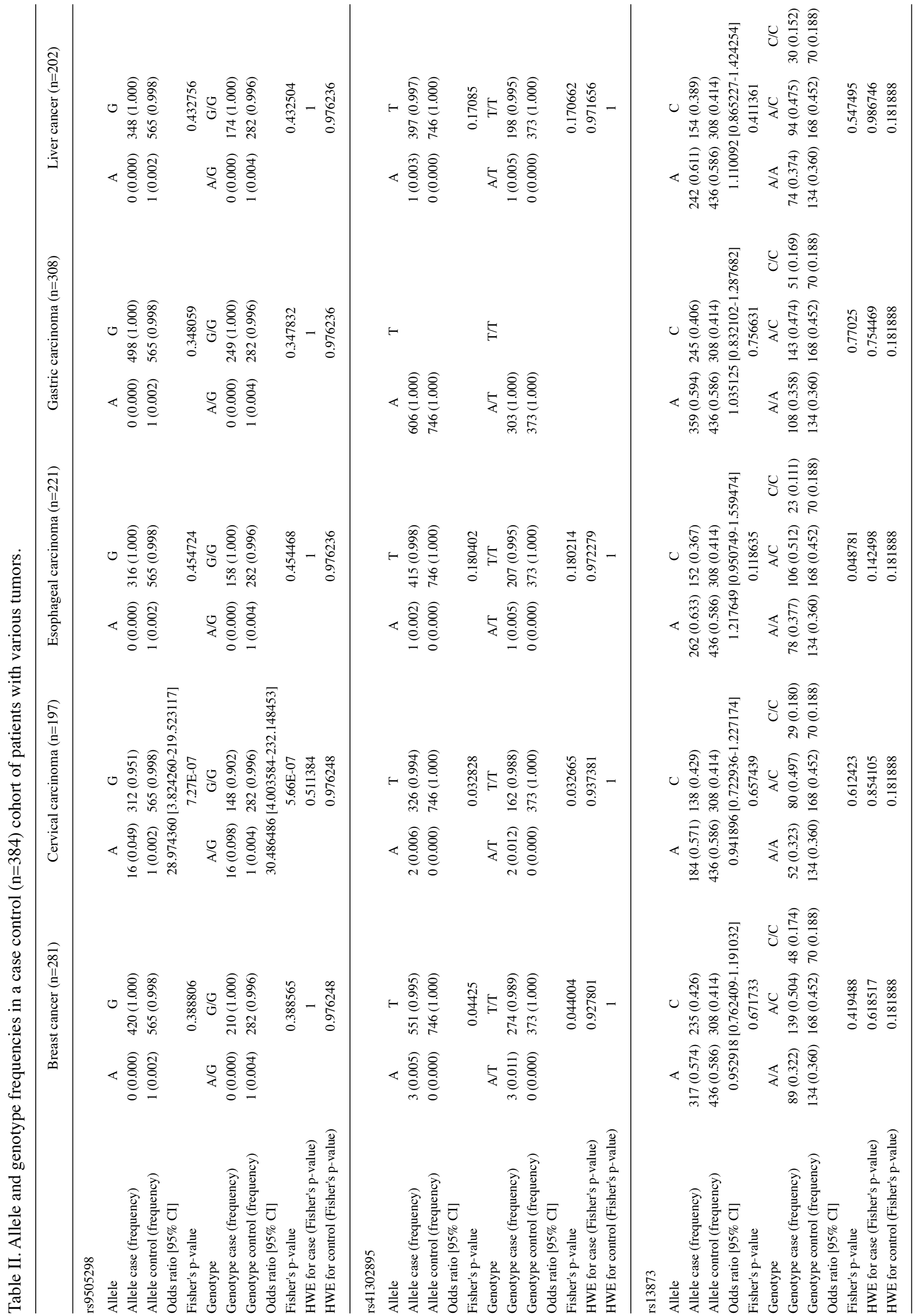




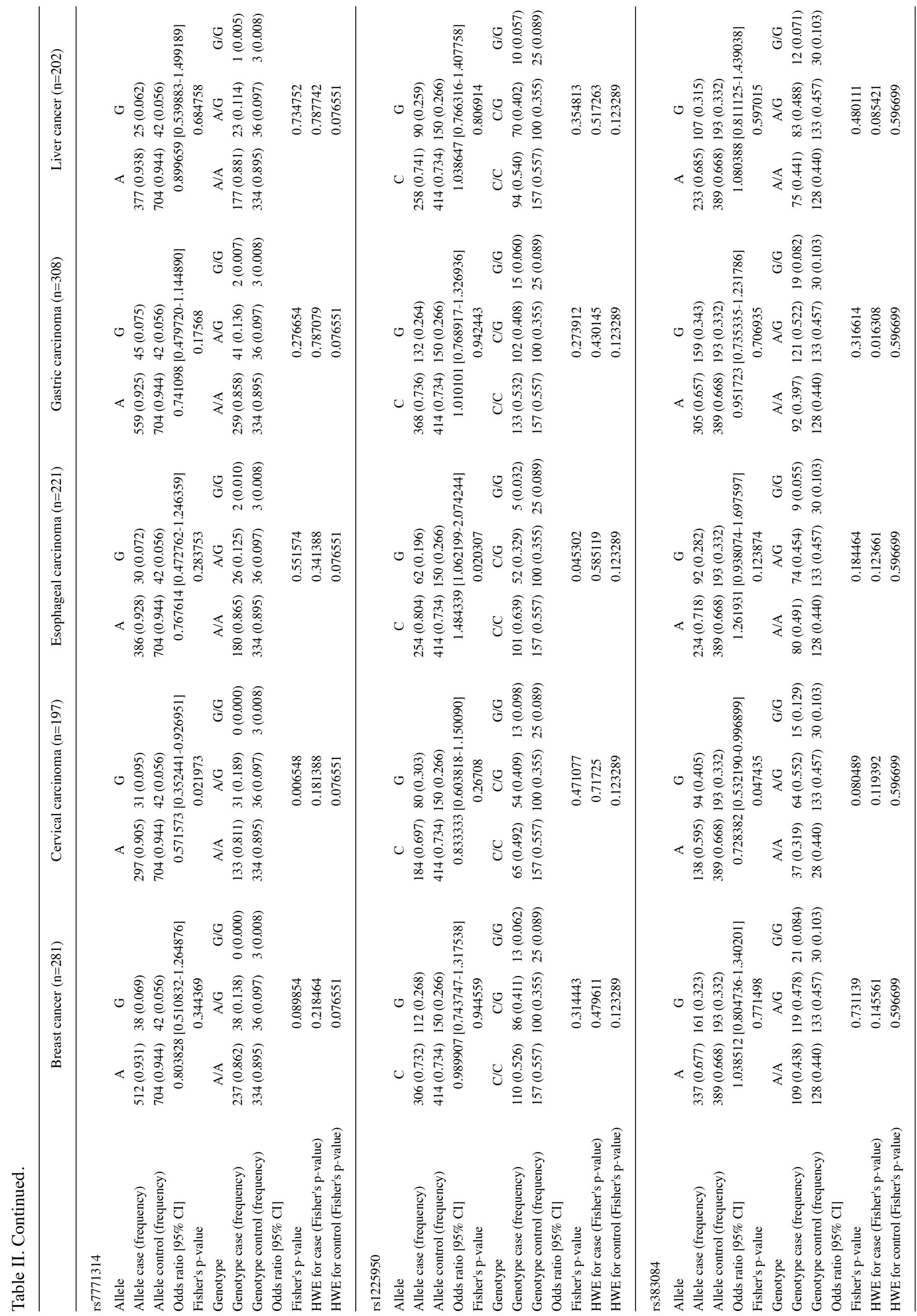




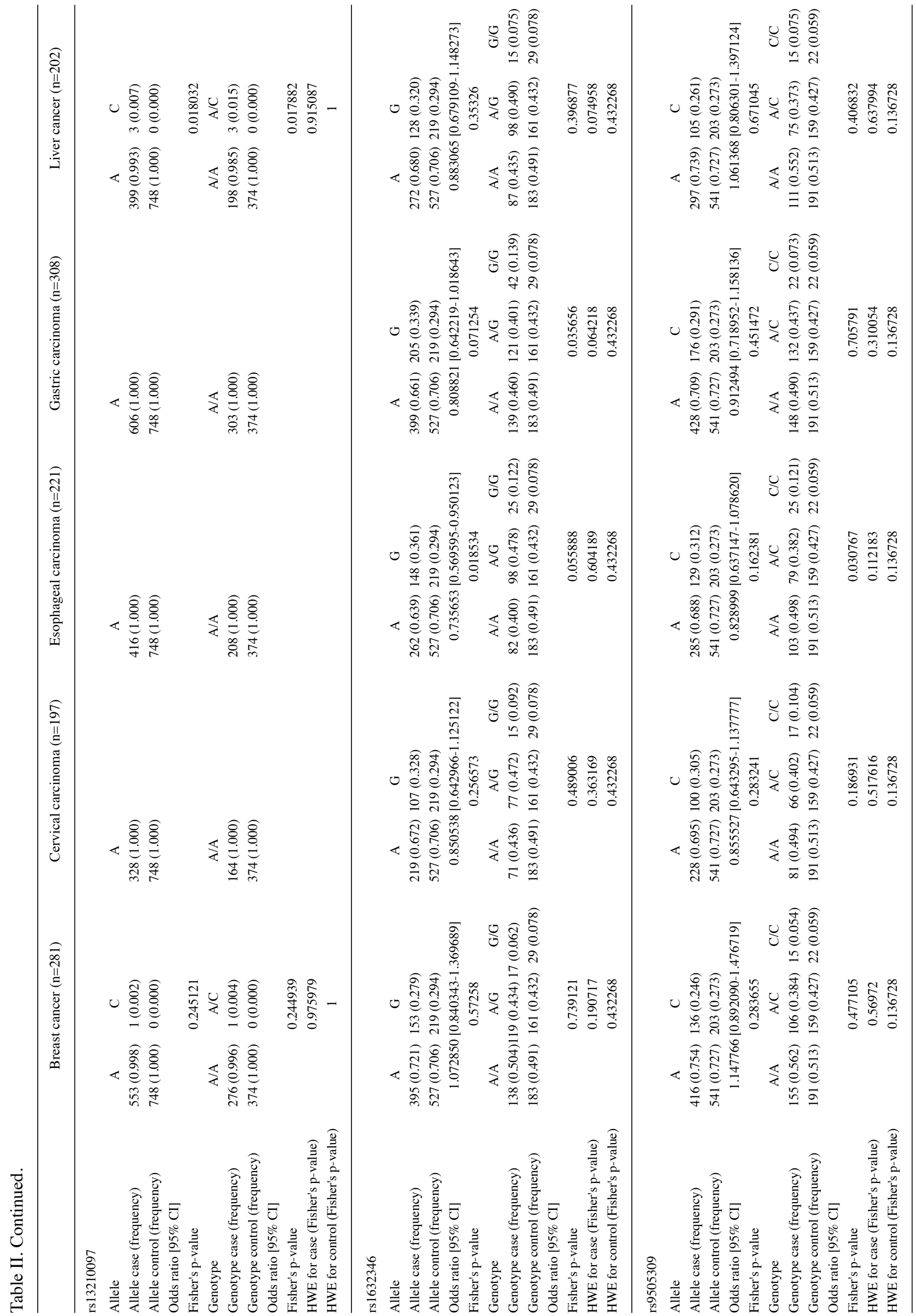




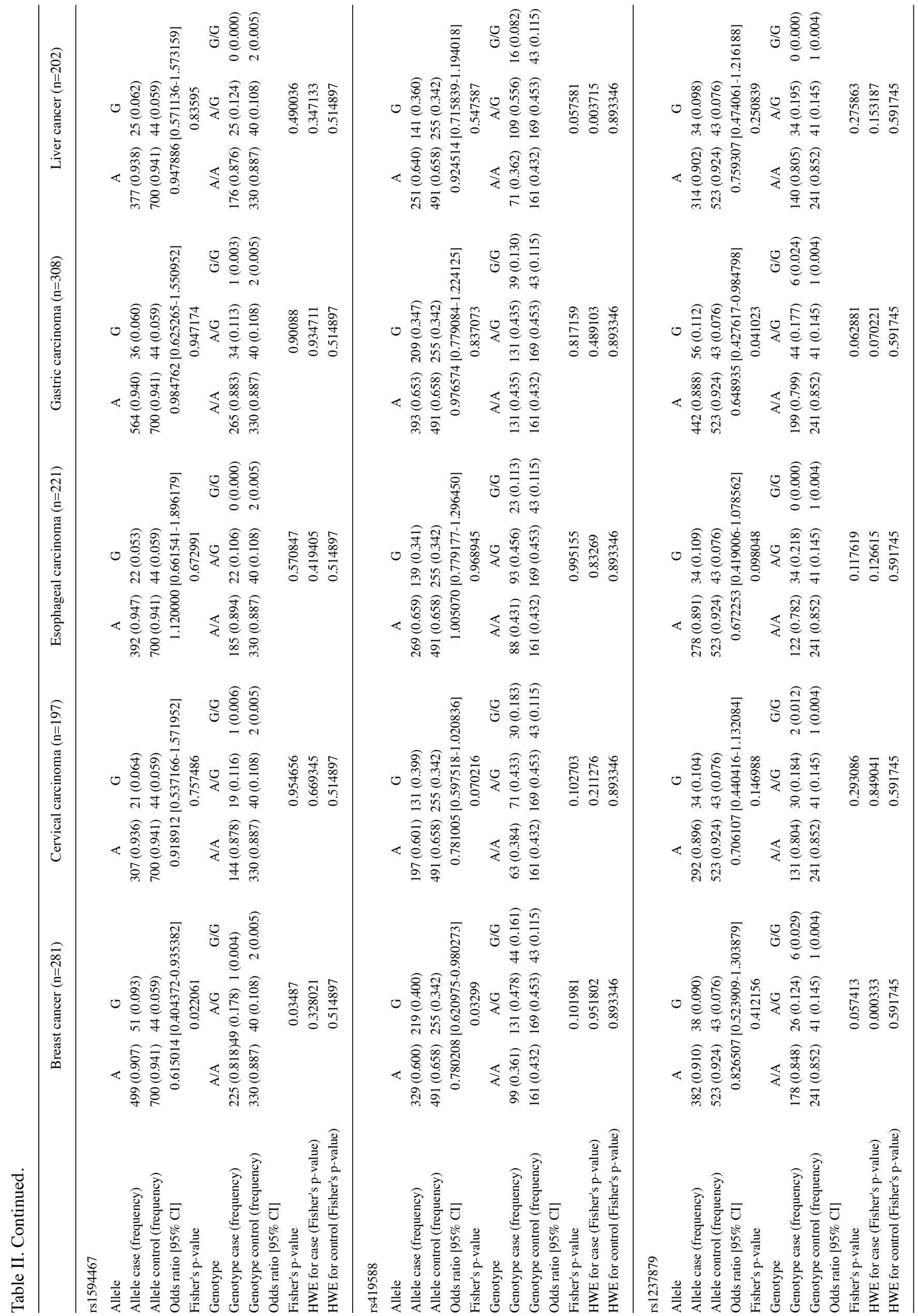




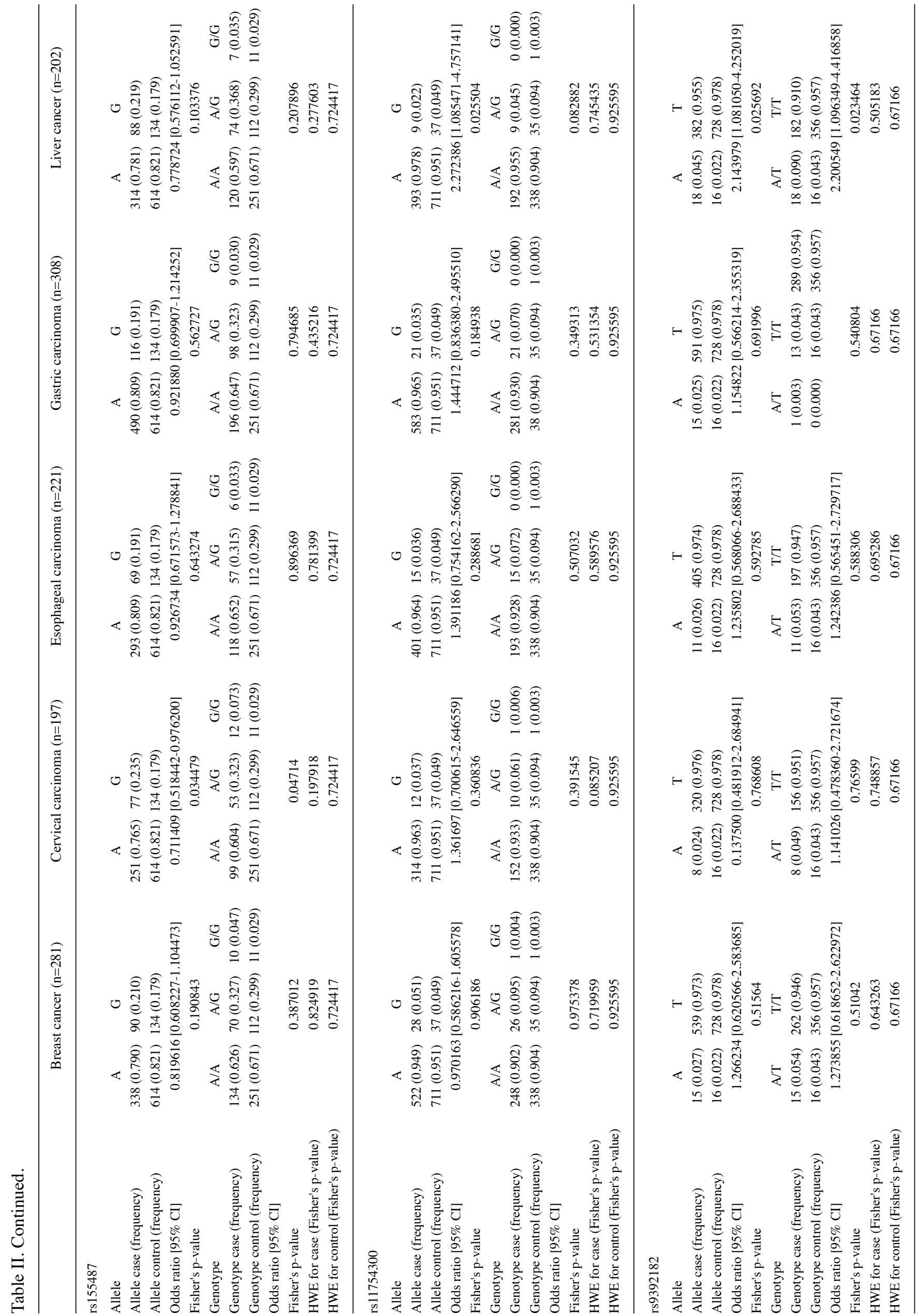




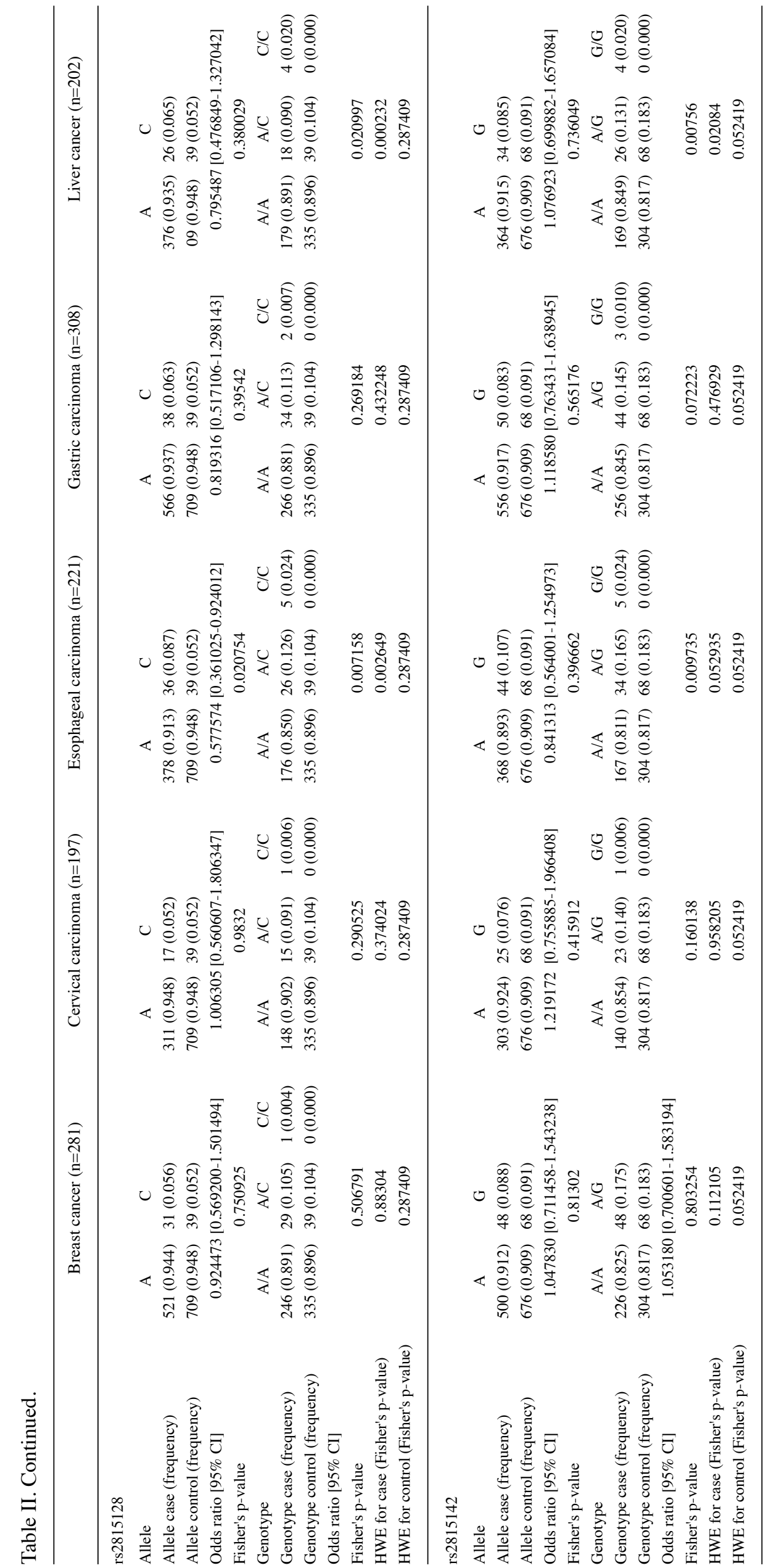


(rs1043784, rs7764128 and rs8643) were statistically associated with non-segmental vitiligo. The haplotypes AGG and GAA, consisting of rs1043784, rs7764128 and rs8643, demonstrated a significant association with the disease (15). Lin et al reported that SNP rs13873 and haplotype rs1225934-rs13873 of BMP6-TXNDC5 genes were significantly associated with schizophrenia (16). We recently found that rs443861 has an association with rheumatoid arthritis using Taqman SNP assay and illumine microassay (17). In the present study, SNPs rs1043784, rs7764128 and rs8643 did not show significant association with tumors. These reports suggest that TXNDC5 contribute to the risk of many diseases.

In conclusion, the present study showed that the expression of TXNDC5 is increased in many tumors. This study also found that TXNDC5 is involved in the proliferation and migration of tumor cells, acting as a tumor-enhancing gene. Moreover, the genetic effect of TXNDC5 was revealed on cervical carcinoma, esophageal carcinoma and liver cancer risk. The present findings are hopefully useful for understanding further the tumorigenic process.

\section{Acknowledgements}

This study was supported by the National Natural Science Foundation of China (NTFC) $(81171990,81373218)$ and the Shandong Taishan Scholarship.

\section{References}

1. Edman JC, Ellis L, Blacher RW, Roth RA and Rutter WJ: Sequence of protein disulphide isomerase and implications of its relationship to thioredoxin. Nature 317: 267-270, 1985.

2. Freedman RB, Hirst TR and Tuite MF: Protein disulphide isomerase: building bridges in protein folding. Trends Biochem Sci 19: 331-336, 1994.

3. Knoblach B, Keller BO, Groenendyk J, Aldred S, Zheng J, Lemire BD, Li L and Michalak M: ERp19 and ERp46, new members of the thioredoxin family of endoplasmic reticulum proteins. Mol Cell Proteomics 2: 1104-1109, 2003.

4. Charlton HK, Webster J, Kruger S, Simpson F, Richards AA and Whitehead JP: ERp46 binds to AdipoR1, but not AdipoR2, and modulates adiponectin signaling. Biochem Biophys Res Commun 392: 234-239, 2010.
5. Sullivan DC, Huminiecki L, Moore JW, Boyle JJ, Poulsom R, Creamer D, Barker J and Bicknell R: EndoPDI, a novel protein-disulfide isomerase-like protein that is preferentially expressed in endothelial cells acts as a stress survival factor. J Biol Chem 47: 47079-47088, 2003.

6. Wang Y, Ma Y, Lü B, Xu E, Huang Q and Lai M: Differential expression of mimecan and thioredoxin domain-containing protein 5 in colorectal adenoma and cancer: a proteomic study. Exp Biol Med (Maywood) 232: 1152-1159, 2007.

7. Vincent EE, Elder DJ, Phillips L, Heesom KJ, Pawade J, Luckett M, Sohail M, May MT, Hetzel MR and Tavaré JML: Overexpression of the TXNDC5 protein in non-small cell lung carcinoma. Anticancer Res 31: 1577-1582, 2011.

8. Zhang L, Hou Y, Li N, Wu K and Zhai J: The influence of TXNDC5 gene on gastric cancer cell. J Cancer Res Clin Oncol 136: 1497-1505, 2010.

9. The International HapMap Consortium: Integrating ethics and science in the International HapMap Project. Nat Rev Genet 5: 467-475, 2004.

10. Barrett JC, Fry B, Maller J and Daly MJ: Haploview analysis and visualization of LD and haplotype maps. Bioinformatics 21: 263-265, 2005.

11. Li Z, Zhang Z, He Z, Tang W, Li T, Zeng Z, He L and Shi Y: A partition-ligation-combination-subdivision EM algorithm for haplotype inference with multiallelic markers: update of the SHEsis. Cell Res 19: 519-523, 2009.

12. Policastro LL, Ibañez IL, Notcovich C, Durán HA and Podhajcer OL: The tumor microenvironment: characterization, redox considerations and novel approaches for ROS-targeted gene therapy. Antioxid Redox Signal 19: 854-895, 2012.

13. Dang CV: Links between metabolism and cancer. Genes Dev 26: 877-890, 2012.

14. Wang L, Zheng Y, Xu H, Yan X and Chang X: Investigate pathogenic mechanism of TXNDC5 in rheumatoid arthritis. PLoS One 8: e53301, 2013.

15. Jeong KH, Shin MK, Uhm YK, Kim HJ, Chung JH and Lee MH: Association of TXNDC5 gene polymorphisms and susceptibility to nonsegmental vitiligo in the Korean population. Br J Dermatol 162: 759-764, 2010.

16. Lin SH, Liu CM, Liu YL, Shen-Jang Fann C, Hsiao PC, Wu JY, Hung SI, Chen $\mathrm{CH}$, Wu HM, Jou YS, Liu SK, Hwang TJ, Hsieh MH, Chang CC, Yang WC, Lin JJ, Chou FH, Faraone SV, Tsuang MT, Hwu HG and Chen WJ: Clustering by neurocognition for fine mapping of the schizophrenia susceptibility loci on chromosome 6p. Genes Brain Behav 8: 785-794, 2009.

17. Chang X,Zhao Y, Yan X, Pan J,Fang K and Wang L: Investigating a pathogenic role for TXNDC5 in rheumatoid arthritis. Arthritis Res Ther 13: R12, 2011 